\title{
Prescription drug use during pregnancy in Southern Tigray region, North Ethiopia
}

Fantahun Molla', Admassu Assen², Solomon Abrha', Birhanetensay Masresha ${ }^{3}$, Arega Gashaw4, Abrham Wondimu', Yared Belete ${ }^{5}$ and Wondim Melkam ${ }^{6 *}$

\begin{abstract}
Background: Judicious utilization of drugs rescues the fetus from the harmful effects while treating the health problems of the pregnant women. This study aimed at evaluating drug utilization pattern and its associated factors among pregnant women in Southern Tigray, Ethiopia.

Method: Institution based cross-sectional study was conducted among 647 pregnant women who had been attending obstetrics-gynecology and antenatal care units in different health facilities of Southern Tigray region. The study participants were selected using multistage sampling technique. Data collection was done using pre-tested semi-structured questionnaires and by reviewing antenatal follow-up cards. Descriptive and inferential statistics were analyzed, to assess drug utilization pattern and its associated factors among pregnant women, using SPSS version 20 software.

Results: Of 647 pregnant women, 87.5\% were prescribed with at least one medication. As per the United States Food and Drug Administration (US-FDA) risk classification system, 87.7, 7.9, 3.9, and 0.5\% of the prescribed drug were from category $A, B, C$ and $D$, respectively. Prescription drug use was more likely among gynecology ward visitors $[A O R=8.97,95 \% \mathrm{Cl}(2.69-29.88)]$ and among those who visited health facilities for the first time during their first $[A O R=2.65,95 \% \mathrm{Cl}(1.44-4.84)]$ and second $[A O R=2.50,95 \% \mathrm{Cl}(1.36-4.61)]$ trimesters.

Conclusion: Majority of the study population used safe and appropriate medications according to US-FDA risk classification system, with the exception of low proportion (0.5\%) of medication with potential risk for the fetus. The average number of drug prescribed per pregnant women was in the recommended range of WHO drug use indicators guideline.
\end{abstract}

Keywords: Pregnancy, Drug use, FDA risk classification, Tigray region

\section{Background}

Ever since the incidence of thalidomide disaster, utilization of medication during pregnancy has become a great concern in health care systems due to the potential fetal risk [1, 2]. However, the reference to avoid all drugs during pregnancy is unrealistic and may endanger both the mother and fetus from complications of untreated acute and chronic medical disorders [3, 4]. Despite its own limitation, judicious selection of drugs based on the United States Food and Drug Administration (FDA) category which classifies drugs into five main categories: A,

\footnotetext{
* Correspondence: melkamwondim@gmail.com

${ }^{6}$ Pharmacology Department, College of Medicine \& Health Sciences, Bahir

Dar University, Bahir Dar, Ethiopia

Full list of author information is available at the end of the article
}

$\mathrm{B}, \mathrm{C}, \mathrm{D}$, and $\mathrm{X}$, is crucial to curb the risk of prescribed drugs to the fetus $[5,6]$.

So far, a significant number of studies have been conducted in various developed $[7,8]$ and developing countries [2, 6, 9-11]. Most of them reported the prescription and consumption of surprisingly a large number of drugs by pregnant women with substantial proportion of drugs from category D and X of US-FDA risk classification system.

In Ethiopia, a few studies have been conducted and reported the use of unsafe medications during pregnancy. Studies in Addis Ababa and Bahir Dar revealed that 71.3 and $88.4 \%$ of the pregnant women used at least one prescription drug of which about 4 and $11 \%$ of them 
received drugs from category $\mathrm{D}$ or $\mathrm{X}$ of the US FDA risk classification, respectively $[6,12]$.

From the preceding studies, there was an excessive consumption of medication by the pregnant women and a considerable number of them were from category $\mathrm{D}$ and $\mathrm{X}$. There is, therefore, a need to know drug utilization trends and associated risk factors among these segments of the community in our country in general and in the region in particular. Moreover, no data is available on the current status of drug utilization pattern among pregnant women in southern Tigray region which the current study aimed to generate.

\section{Methods}

\section{Study area and design}

An institution based cross-sectional study was conducted, in February 2015, among pregnant women who had been receiving services in obstetrics-gynecology and antenatal care (ANC) units in the selected health centers and hospital in Southern Tigray region. There were a total of 39 health facilities (35 health centers and 4 hospitals) in the study area. The study area is one of seven administrative zones of the Regional State of Tigray. Mekelle, the capital city of the region, is $783 \mathrm{~km}$ away from Addis Ababa, the capital city of Ethiopia. According to the 2007 Census conducted by the Central Statistical Agency of Ethiopia [13], the region has an estimated total population of $4,314,456$, of which $2,124,853$ were men and 2,189,603 were women; urban inhabitants account 842,723 or $19.53 \%$ of the population.

\section{Sample size determination and sampling procedure}

A single population proportion formula was used to calculate the sample size. Using the $41 \%$ prevalence of drug exposure of pregnant women in Mekelle city [14], 5\% margin of error at $95 \%$ confidence level, a design effect of 2 , and $10 \%$ inclusion of non-response rate, the calculated final sample size was 709. Multistage sampling technique was employed to select the health facilities. There were 35 health centers and 4 hospitals in the study area. In stage I, 20\% from each stratum (health center and hospital) was included in the study to ensure the representativeness of the sample. Accordingly, six health centers (Adishehu, Betemera, Korem, kukuftu, Alamata, Timuga) and one hospital (Michew hospital) were selected by lottery method. In stage II, the number of study participants from each study site was determined using the proportion to population size (probability proportional to size) method and in stage III the samples determined in stage II were allocated to each selected health facilities using simple random sampling.

\section{Data collection and analysis}

Data were collected using pre-tested semi-structured questionnaires and by reviewing antenatal follow-up cards of pregnant women. The semi-structured questionnaire was employed to collect socio-demographic data, obstetric and medical history of pregnant women. The ANC chart was reviewed to evaluate drugs which had been prescribed previously. The data extraction forms were used to collect information on the total number of ANC visits, gestational age, and drugs prescribed during each trimester. Data on medication were not collected for the entire pregnancy but for the trimesters at which the pregnant women were at the time of data collection. Data were collected by experienced nurses who had been working in ANC rooms.

Quantitative data were cleaned and entered using EPIINFO version 3.1 .5 and analyzed by SPSS version 20 software. Contingency tables were created to assess frequencies, and percentages of the variables were done in order to describe them in relation to the study population. The odds ratio was used to assess the association between dependent and independent variables. Variables which showed a statistically significant association $(P<0.05)$ in bivariate analysis were considered for multivariate model.

\section{Ethical considerations}

Ethical approval and clearance were obtained from the Ethical Review Committees of both the College of Health Sciences of Mekelle University and Tigray Regional Health Bureau. At all levels, officials were contacted and permission was secured. The purpose of the study was explained to the study participants, confidentiality was ensured and verbal consent was obtained before data collection.

\section{Results}

Of the total 709 questionnaires distributed to be filled by the data collectors, 647 were filled completely which gives a response rate of $91.3 \%$. The remaining $62(8.7 \%)$ questionnaires were excluded from the analysis for gross incompleteness and inconsistency of responses.

\section{Socio-demographic characteristics}

Socio- demographic characteristics of pregnant women in Southern Tigray region is presented in Table 1. Most of the pregnant women $(59.2 \%)$ were in the age group of $18-25$ years with the mean age of $24.88 \pm 5.17$ years. Majority of the study participants were Orthodox Christian (80.4\%) and married (95.8\%). Regarding residency, more than half of them (57.8\%) were urban dwellers, and $46 \%$ of them attended formal education. 
Table 1 Socio-demographic characteristics of pregnant women in Southern Tigray region, Ethiopia, February $2015(n=647)$

\begin{tabular}{lll}
\hline Variable & Frequency (\%) & Percentage (\%) \\
\hline Age group & 383 & 59.2 \\
$18-25$ & 201 & 31.1 \\
$26-30$ & 37 & 5.7 \\
$31-35$ & 26 & 4.0 \\
$36-40$ & & \\
Marital status & 16 & 2.5 \\
Single & 620 & 95.8 \\
Married & 11 & 1.7 \\
Divorced & & \\
Educational status & 345 & 53.3 \\
Illiterate & 163 & 25.2 \\
Primary education & 98 & 15.1 \\
Secondary education & 41 & 6.3 \\
Tertiary education & & \\
Employment status & 111 & 17.2 \\
Employed & 536 & 82.8 \\
Unemployed & 374.8 \\
Residency & 273 & \\
Urban & & \\
Rural & & \\
\hline
\end{tabular}

\section{Obstetrics and medical information}

Most of the pregnant women (84.7\%) visited the health facilities to attend ANC service and the remaining visited obstetrics- gynecology ward (Table 2). Concerning the time of their first visit,34.2, 35.2 and $30.6 \%$ pregnant women visited the health facilities for the first time during the first, second and third trimesters of their current pregnancy, respectively. Majority (71.9\%) of the women visited the service centers for a maximum of 2 times (12 times). The main reason for their visit was seeking ANC services (96.9\%). Over half (61.5\%) of the pregnant women were multigravida, and $88.9 \%$ of them were planned pregnancies. Apart from 2.3\% HIV positive pregnant women, none of the respondents reported any kind of chronic disease. Among 440 pregnant women who had hemoglobin test, $4.1 \%$ were anemic. Only three women had a history of hospitalization during their current pregnancy. Forty-seven respondents had a history of either spontaneous $(80.9 \%)$ or induced $(19.1 \%)$ abortion.

\section{Drug use characteristics}

A total of $566(87.5 \%)$ pregnant women were prescribed with 634 drugs including supplements, (Table 3). The average number of drug prescribed per pregnant women was 1.1. Almost all (98.2\%) of those prescribed with
Table 2 Pregnancy-related variables of respondents in Southern Tigray region, Ethiopia, February $2015(n=647)$

\begin{tabular}{|c|c|c|}
\hline Variable & Frequency & Percentage (\%) \\
\hline \multicolumn{3}{|l|}{ Gravidity } \\
\hline Primigravida & 249 & 38.5 \\
\hline Multigravida & 398 & 61.5 \\
\hline \multicolumn{3}{|l|}{ Pregnancy status } \\
\hline Planned & 575 & 88.9 \\
\hline Mistimed & 53 & 8.2 \\
\hline Unwanted & 19 & 2.9 \\
\hline \multicolumn{3}{|c|}{ Number of visit to health facilities } \\
\hline $1-2$ times & 465 & 71.9 \\
\hline 3-4 times & 178 & 27.5 \\
\hline$\geq 5$ times & 4 & 0.6 \\
\hline \multicolumn{3}{|c|}{ Time of first visits of the health facilities } \\
\hline First trimester & 221 & 34.2 \\
\hline Second trimester & 228 & 35.2 \\
\hline Third trimester & 198 & 30.6 \\
\hline \multicolumn{3}{|c|}{ Reasons for visit to the health facilities } \\
\hline ANC & 627 & 96.9 \\
\hline Illness & 19 & 2.9 \\
\hline Other & 1 & 0.2 \\
\hline \multicolumn{3}{|l|}{ Visited ward } \\
\hline ANC & 548 & 84.7 \\
\hline Gynecology & 99 & 15.3 \\
\hline \multicolumn{3}{|c|}{ Hospitalization during pregnancy } \\
\hline No & 644 & 99.5 \\
\hline Yes & 3 & 0.5 \\
\hline \multicolumn{3}{|l|}{ Chronic illness } \\
\hline No & 647 & 97.7 \\
\hline Yes & 15 & 2.3 \\
\hline \multicolumn{3}{|l|}{ Abortion } \\
\hline No & 600 & 92.7 \\
\hline Yes & 47 & 7.3 \\
\hline \multicolumn{3}{|l|}{ Type for abortion } \\
\hline Spontaneous abortion & 38 & 80.9 \\
\hline Induced abortion & 9 & 19.1 \\
\hline \multicolumn{3}{|l|}{ Hemoglobin level } \\
\hline Anemic & 18 & 4.1 \\
\hline Normal & 422 & 95.9 \\
\hline \multicolumn{3}{|l|}{ Alcohol drinking } \\
\hline No & 646 & 99.8 \\
\hline Yes & 1 & 0.2 \\
\hline \multicolumn{3}{|l|}{ Smoking } \\
\hline No & 646 & 99.8 \\
\hline Yes & 1 & 0.2 \\
\hline \multicolumn{3}{|l|}{ Khat use } \\
\hline No & 637 & 98.5 \\
\hline Yes & 10 & 1.5 \\
\hline
\end{tabular}


Table 3 Medications used by pregnant women at different trimester and their US FDA risk classification in Southern Tigray region, Ethiopia, February $2015(n=634)$

\begin{tabular}{lllllll}
\hline Prescribed drugs & 1st trimester & 2nd trimester & 3rd trimester & Total & Drug class & FDA class \\
\hline Iron/folate & 221 & 228 & 87 & 536 & Supplements \\
Multivitamins & 7 & 10 & 12 & 29 & Supplements & A \\
Amoxicillin & 2 & - & 10 & 24 & Antibiotics \\
Metronidazole & - & - & 1 & 1 & Antiprotozoal & B \\
Clotrimazole & - & 2 & 2 & 2 & Antifungal \\
Antacid & 3 & 6 & 4 & 9 & Drug for PUD* & C \\
Paracetamol & 2 & - & 7 & 15 & Analgesic \\
Cotrimoxazole & - & 6 & 1 & 1 & Antibiotics \\
TDF/3TC/EFV & 8 & 1 & 1 & 15 & Antiviral & D \\
Cephalexin & 2 & - & 2 & 3 & Antibiotics \\
Diclofenac & 1 & 1 & 2 & 1 & Analgesic \\
Ceftriaxone & - & - & - & 1 & Antibiotics \\
Metoclopramide & 1 & - & - & 1 & Antiemetic \\
Benzathine penicillin & 1 & & & Antibiotics
\end{tabular}

*PUD- Peptic Ulcer Disease

drugs had received supplemental drugs. Iron/folic acid (95\%) was the most commonly prescribed of supplements; the remaining $5 \%$ was multivitamins.

Of the total 634 prescribed drugs, 78 (12.3\%) were nonsupplemental drugs: antibacterial, antiviral, antifungal, analgesics, antiemetic and others; the dominant prescription being antibiotics followed by analgesics (Table 3). More than two third of the non-supplemental drugs were prescribed during the second (36\%) and third (38.5\%) trimesters.

According to the FDA pregnancy risk category for prescription drugs, the present study showed that 87.7, 7.9, 3.9 , and $0.5 \%$ of the drugs belongs to risk classification $\mathrm{A}, \mathrm{B}, \mathrm{C}$ and $\mathrm{D}$, respectively.

\section{Factors associated with pregnant women's exposure to prescribed drugs}

From the bivariate regression analysis, the type of ward, numbers of visits to the health facilities, trimester at the first visit, and gravidity were significantly associated with exposure to prescribed drugs (Table 4). These independent variables were further considered for multivariate analysis. Accordingly, multigravida pregnant women were less likely to be prescribed with the drugs as compared to primigravida $[\mathrm{AOR}=0.43,95 \% \mathrm{Cl}(0.24-0.78)]$. Similarly, those pregnant women with a 3-4 times visit had less chance of being exposed to drugs in comparison to those pregnant women who had 1-2 times visit $[\mathrm{AOR}=0.50,95 \% \mathrm{Cl}(0.29-0.85)]$. However, those who sought medical help in gynecology ward were found to be 9 times more likely to have a prescribed drug as compared to those who visited ANC ward [AOR = 8.97, 95\% $\mathrm{Cl}$ (2.69-29.88)]. Pregnant women who visited the wards for the first time during the first $[\mathrm{AOR}=2.65,95 \% \mathrm{Cl}$ $(1.44-4.84)$ ] and second $[\mathrm{AOR}=2.50,95 \% \mathrm{Cl}(1.36-$ 4.61)] trimesters were with high odds of being prescribed with drugs.

\section{Discussion}

Prescribing drugs during pregnancy presents a challenge to physicians as pregnant women must be treated while protecting the fetuses against possible side effects of the drugs [15]. This would in turn be dependent on the proportion of drugs received by pregnant women during the

Table 4 Factors associated with exposure to prescribed drugs among pregnant women in Southern Tigray region, Ethiopia, February 2015

\begin{tabular}{lccc}
\hline Variable & COR $(95 \% \mathrm{Cl})$ & AOR $(95 \% \mathrm{Cl})$ & $P$-value \\
\hline $\begin{array}{l}\text { Gravidity } \\
\text { Primigravida }\end{array}$ & 1 & & \\
$\begin{array}{l}\text { Multigravida } \\
\text { Number of visits to health facilities }\end{array}$ & & & \\
1-2 times & 1 & & \\
3-4 times & $0.40(0.24-0.65)$ & $0.50(0.29-0.85)$ & 0.011 \\
$\geq 5$ times & $0.10(0.01-0.74)$ & $0.15(0.01-1.33)$ & 0.090 \\
Ward & 1 & & \\
ANC & & & \\
Gynecology & $5.31(1.64-17.17)$ & $8.97(2.69-29.88)$ & 0.001 \\
First visit to the health facility & & \\
First trimester & $2.62(1.47-4.65)$ & $2.64(1.44-4.84)$ & 0.002 \\
Second trimester & $2.71(1.53-4.81)$ & $2.50(1.36-4.61)$ & 0.003 \\
Third trimester & 1 & & \\
\hline COD: crude odds ratio; AOD: Adjusted odds ratio &
\end{tabular}


course of their gestational period. The prevalence of drug utilization during pregnancy in this study was found to be $87.5 \%$. This value is higher as opposed to the study conducted in United States, Italy and Ethiopia that reported 82, 75 and $71.3 \%$, respectively $[7,12,16]$, but comparable with the report of WHO which is $86 \%$ [17]. The observed higher proportion of the drug utilization in this study could be partly attributed to the supplements prescribed as $98.2 \%$ of the pregnant women who were prescribed with medication received supplements in the current study. From the supplement categories, iron/folic acid (95\%) was the most frequently received supplement in the present study which is an appealing finding when it is compared to a study conducted in eight rural districts of Ethiopia where only $35.4 \%$ of the women who gave birth in the preceding year were prescribed with iron tablets [18].

The most frequently prescribed non-supplemental drugs were antibiotics (41\%) followed by analgesics (23\%) which is in agreement with the previous studies done in Ethiopia [19, 20]. Antibiotics are one of the most frequently prescribed medications during pregnancy with estimated range of 19 to 44\% [21]. Paracetamol was the most widely used analgesic and antipyretic drug in this study and higher utilization was observed in the third trimester.

The US-FDA pregnancy risk classification system was used to evaluate the risk levels of drugs prescribed during pregnancy. The majority of prescribed drugs in the present study were safe as 87.7 and $7.9 \%$ were found to be category A and B, respectively. Only three pregnant women $(0.5 \%)$ were prescribed from category D drugs in the current study, which is by far lower as compared to the findings of the other studies $[6,19,20]$. However, even a small increase in the risk of birth defects may have significant implications for public health. This result emphasizes the need for thorough consideration while prescribing drugs to pregnant women. The drugs which were prescribed from category $\mathrm{D}$ were diclofenac (when prescribed at third trimester) and cotrimoxazole. As opposed to different studies $[6,19,20]$ conducted in other parts of Ethiopia, where $0.4-11 \%$ of pregnant women was prescribed with drugs in category $\mathrm{X}$, there was not a single pregnant woman who was prescribed with category $\mathrm{X}$ drugs in our study.

Multigravida pregnant women were less likely to be prescribed with drugs as compared to primigravida in the current study. The reason might be multigravida pregnant women would have tolerated some of the physiological changes which prompt drugs use in the case of primigravida woman. This finding is in contrast to the study conducted in Bahir Dar city Administration [6].
Those pregnant women having 3-4 times visit had less chance of being exposed to drugs in comparison to those who had 1-2 times visit. A similar trend of less drug prescription with increased number of visits to the health facilities was observed in the study conducted in Brazil [22]. This could be due to the fact that the more frequent the visiting the better is the counseling of the health providers for the pregnant women regarding the issues related to different aspects of pregnancy. This might have helped the pregnant women to manage their health problem without actually consuming drugs. In contrast, other studies reported the utilization of more drugs by the pregnant woman with more frequent prenatal visits $[22,23]$.

Pregnant women who had their first visit during the first and second trimester had high odds of being prescribed with drugs. This may be attributed to the fact that most of the health providers are accustomed to give supplements in the first and second trimester and the majority of drugs prescribed in this study were supplements. .

Pregnant women who sought medical help in gynecology ward were more likely to have a prescribed drug as compared to those who visited ANC ward. This finding is in accordance with the study done in Ayder referral hospital, Ethiopia, [14] where the highest average number of medications prescribed per pregnant woman was recorded in the gynecology ward.

The main limitation of this study was a temporal relationship establishment with some variables, the crosssectional design employed in this study which could not provide much more substantial evidence of causality as compared to longitudinal design. Moreover, we did not examine over-the-counter medications and drugs dispensed in the hospital. It is possible that this could underestimate the prevalence of drug utilization particularly for drugs that are commonly available over the counter.

\section{Conclusions}

Majority of the study population used safe and appropriate medications according to US-FDA risk classification system, with the exception of low proportion $(0.5 \%)$ of medication with potential risk for the fetus. The average number of drugs prescribed per pregnant women was in the recommended range of WHO drug use indicators guideline. Even though the drug utilization pattern in this study is encouraging, the issue of prescribing drugs form category D and X should be addressed by informing the prescribers to stick to the treatment guideline. Besides, educating and counseling pregnant women and women of child bearing ages regarding the safety of drugs during pregnancy are important to reduce the risk on the fetus. 


\section{Acknowledgements}

We would like to thank data collectors and study participants for their invaluable time.

\section{Funding}

This research was funded by Mekelle University from recurrent budget. The funding body had no role in the design of the study, data collection, analysis, interpretation of data, and writing the manuscript.

\section{Availability of data and materials}

Data used in this analysis are not publicly available, as they are confidential. However, the data will be accessed for research purpose by contacting the corresponding author through the following email address melkamwondim@gmail.com.

\section{Authors' contributions}

Conceived and designed the experiments: FM, AA and WM. Performed the experiments: FM, AA, AG, AW, BM, SA, WM and YB. Analyzed the data: FM, AA and WM. Wrote the paper: FM, AA, AG, AW, BM, SA, WM and YB. All authors have read and approved the final manuscript.

\section{Competing interests}

The authors have declared that there are no competing interests.

\section{Consent for publication}

Not applicable.

\section{Ethics approval and consent to participate}

This research was approved by health research ethics review committee of college of health sciences, Mekelle University. All the participants were informed about the aim of the study, and the relevance of their participation for the study. They were told about the confidentiality of the information they provided. Information was also provided to every participant about the right to refuse to participate, to stop the interview at any time, and to skip any question he/she doesn't like to reply. Finally, the interview was made after getting their verbal consent.

\section{Publisher's Note}

Springer Nature remains neutral with regard to jurisdictional claims in published maps and institutional affiliations.

\section{Author details}

'Pharmaceutics Department, School of Pharmacy, College of Health Sciences, Mekelle University, Mek'ele, Ethiopia. ${ }^{2}$ Department of Pharmacy, College of Medicine and Health Sciences, Wollo University, Dessie, Ethiopia.

${ }^{3}$ Pharmacology and Toxicology Department, School of Pharmacy, College of Health Sciences, Mekelle University, Mek'ele, Ethiopia. ${ }^{4}$ Clinical Pharmacy Unit, School of Pharmacy, College of Health Sciences, Mekelle University, Mek'ele, Ethiopia. ${ }^{5}$ Social Pharmacy and Pharmacoepidemiology Unit, School of Pharmacy, College of Health Sciences, Mekelle University, Mek'ele, Ethiopia. ${ }^{6}$ Pharmacology Department, College of Medicine \& Health Sciences, Bahir Dar University, Bahir Dar, Ethiopia.

Received: 26 September 2016 Accepted: 26 May 2017 Published online: 05 June 2017

\section{References}

1. Al-Riyami IM, Al-Busaidy IQ, Al-Zakwani IS. Medication use during pregnancy in Omani women. Int J Clin Pharm. 2011;33:634-41.

2. Abubakar K, Abdulkadir R, Abubakar SB, Jimoh AO, Danzaki AM. Drug utilization pattern in pregnancy in a Tertiary Hospital in Sokoto, North West. J Heal Sci. 2014;4(4):99-104.

3. Ezeui EAE, Oparah AC, Enato E. Assessment of prescription profile of pregnant women visiting antenatal clinics. Pharm Pract. 2007;5(3):135-9.

4. Etefa K, Kahissay MH. Assessment of drug prescribing pattern among pregnant women attending antenatal Care in Health Centers found in Arada Subcity, Addis Ababa, Ethiopia. J Pharm Sci Bioscientific Res. 2015;5:347-62.

5. Araújo DD, Leal MM, Jucielly E, Santos V, Leal LB. Consumption of medicines in high-risk pregnancy: evaluation of determinants related to the use of prescription drugs and self-medication. Braz J Pharm Sci. 2013;49(3):492-505.

6. Admasie C, Wasie B, Abeje G. Determinants of prescribed drug use among pregnant women in Bahir Dar city administration, Northwest Ethiopia: a cross sectional study. BMC Pregnancy Childbirth. 2014;14:325.

7. Andrade S, Gurwitz J, Davis R, Chan K, Finkelstein J, Fortman K, et al. Prescription drug use in pregnancy. Am J Obst Gynecol. 2004;191(2): 398-407.

8. Bakker M, Jentink J, Vroom F, Van Den Berg P, De Walle H, De Jong-Van Den Berg L. Drug prescription patterns before, during and after pregnancy for chronic, occasional and pregnancy-related drugs in the Netherlands. Bjog. 2006;113(5):559-68

9. Hanafy SA, Sallam SA, Kharboush IF, Wahdan IH. Drug utilization pattern during pregnancy in Alexandria, Egypt. Eur J Pharm Med Res. 2016;3(2): 19-29.

10. Gawde SR, Bhide SS, Patel TC, Chauhan AR, Mayadeo NM. Drug prescription pattern in pregnant women attending antenatal out patient Department of a Tertiary Care Hospital. Br J Pharm res. 2013;3(1):1-12.

11. Cleary BJ, Butt H, Strawbridge JD, Gallagher PJ, Fahey T, Murphy DJ. Medication use in early pregnancy-prevalence and determinants of use in a prospective cohort of women. Pharmacoepidemiol Drug Saf. 2010; 19(4):408-17.

12. Kebede B, Gedif T, Getachew A. Assessment of drug use among pregnant women in Addis Ababa, Ethiopia. Pharmacoepidemiol Drug Saf. 2008;18:1-12

13. CSA. Population and housing census report-Tigray region - 2007. In: Central Statistical Agency, 2010-07; 2007. http://www.csa.gov.et/index.php/2013-0220-14-51-51/2013-04-01-11-53-00/census-2007 (Accessed on 01 Aug 2016).

14. Gebreegziabher TL, Berhe DF, Gutema GB, Kabtyimer BN. Drug utilization pattern and potential teratogenesity risk among pregnant women; the case of Ayder referral hospital, Tigray -Ethiopia. IJPSR. 2012;3(5):1371-8.

15. Stokholm J, Schjørring S, Pedersen L, Bischoff AL, Følsgaard N, Carson CG, et al. Prevalence and predictors of antibiotic administration during pregnancy and birth. PLoS One. 2013;8(12):1-7.

16. Donati S, Baglio G, Spinelli A, Grandolfo ME. Drug use in pregnancy among Italian women. Eur J Clin Pharmacol. 2000;56(4):323-8.

17. Briggs GG, Freeman RK, Yaffe SJ. Drugs in pregnancy and lactation. A reference guide to fetal and neonatal risk. 10th ed. Philadelphia : Wolters Kluwer/Lippincott Williams \& Wilkins Health; 2015.

18. Gebremedhin S, Samuel A, Mamo G, Moges T, Assefa T. Coverage, compliance and factors associated with utilization of iron supplementation during pregnancy in eight rural districts of Ethiopia: a cross-sectional study. BMC Public Health. 2014;14:607.

19. Gebremedhin L, Gomathi P. Assessment of drug use and effect in pregnant women attending antenatal care in hospitals of Mekelle, Tigray, Ethiopia. J Drug Deliv Ther. 2014;4(6):75-82.

20. Mohammed MA, Ahmed JH, Bushra AW, Aljadhey HS. Medications use among pregnant women in Ethiopia: a cross sectional study. J Appl Pharm Sci. 2013;3(04):116-23

21. Meeraus WH, Petersen I, Gilbert R. Association between Antibiotic Prescribing in Pregnancy and Cerebral Palsy or Epilepsy in Children Born at Term: A Cohort Study Using The Health Improvement Network. PLoS ONE. 2015;10(3):e0122034. doi:10.1371/journal.pone.0122034.

22. Fontoura A, Ayres LR, Martins-Nagai M, Dewulf NLS, Dos Santos V, Martinez EZ, et al. Prevalence of medication use among low risk pregnant women: a drug utilization study. Afr J Pharm Pharmacol. 2014;8(36):883-92.

23. Brum LF, Pereira P, Felicetti LL, Da Silveira RD. Prescribed and unprescribed drug use among pregnant patients attended by the unified health system in Santa Rosa (state of Rio Grande do Sul, Brazil). Cien Saude Colet. 2011; 16(5):2435-42 